\title{
Noise-driven multistability versus deterministic chaos in phenomenological semi-empirical models of whole-brain activity
}

\author{
Juan Piccinini, ${ }^{1, \text { a) }}$ Ignacio Perez Ipina, ${ }^{1}$ Helmut Laufs, ${ }^{2}$ Morten Kringelbach, ${ }^{3}$ Gustavo Deco, ${ }^{4}$ Yonatan Sanz \\ Perl, ${ }^{1,4,5}$ and Enzo Tagliazucchi ${ }^{1}$ \\ 1)Buenos Aires Physics Institute and Physics Department, University of Buenos Aires, Buenos Aires 1428, \\ Argentina \\ ${ }^{2)}$ Neurology Department, University of Kiel, Kiel 24105, Germany \\ 3) Department of Psychiatry, University of Oxford, Oxford OX3 7JX, United Kingdom \\ 4) Center for Brain and Cognition, Computational Neuroscience Group, Universitat Pompeu Fabra, Barcelona 08002, \\ Spain \\ 5) Universidad de San Andrés, Victoria B1644BID, Argentina
}

(Dated: 10 May 2021)

An outstanding open problem in neuroscience is to understand how neural systems are capable of producing and sustaining complex spatiotemporal dynamics. Computational models that combine local dynamics with in vivo measurements of anatomical and functional connectivity can be used to test potential mechanisms underlying this complexity. We compared two conceptually different mechanisms: noise-driven switching between equilibrium solutions (modeled by coupled Stuart-Landau oscillators) and deterministic chaos (modeled by coupled Rossler oscillators). We found that both models struggled to simultaneously reproduce multiple observables computed from the empirical data. This issue was especially manifest in the case of noise-driven dynamics close to a bifurcation, which imposed overly strong constraints on the optimal model parameters. In contrast, the chaotic model could produce complex behavior over a range of parameters, thus being capable of capturing multiple observables at the same time with good performance. Our observations support the view of the brain as a non-equilibrium system able to produce endogenous variability. We presented a simple model capable of jointly reproducing functional connectivity computed at different temporal scales. Besides adding to our conceptual understanding of brain complexity, our results inform and constraint the future development of biophysically realistic large-scale models.

The quote "What I cannot create, I do not understand" was found written in the blackboard of celebrated physicist Richard Feynman at the time of his death. This sentence suggests a way forward for neuroscientists interested in unravelling the principles behind the richness and complexity of spontaneous brain dynamics. Over the last decades, tremendous advances in neuroimaging enabled the construction of whole-brain activity models with real predictive power in the statistical sense. It is now possible to create realistic complex dynamics, instead of passively screening for their presence in neuroimaging data. We contrasted two different types of building blocks (i.e. two choices of local dynamics) and tested their capacity to reproduce the empirical data, with the purpose of increasing our conceptual understanding of the mechanisms behind large-scale spontaneous activity in the human brain.

\section{INTRODUCTION}

Since the mid 1990s, the spontaneous large-scale ${ }^{1}$ dynamics of the human brain have attracted a growing body of research ${ }^{2}$. Previously disregarded as experimental noise, it became increasingly clear that the fluctuating dynamics of endogenous brain activity were not random, but highly structured in the spatial and temporal domains ${ }^{3,4}$. During rest, this

\footnotetext{
${ }^{a)}$ Electronic mail: piccijuan@gmail.com
}

activity self-organizes into recurrent patterns that overlap with different functional systems of the brain, known as resting state networks $(\mathrm{RSN})^{5}$. Since the activity patterns evoked by cognitive tasks and sensory stimulation can be put into correspondence with the $\mathrm{RSN}^{6}$, it has been proposed that the spontaneous dynamics of the brain reflect a metastable exploration of states that facilitate rapid reactivity upon environmental demands $^{3,7-9}$. According to this view, evolution has shaped the brain as an itinerant dynamical system which is always close to configurations associated with sensory, cognitive or motor functions ${ }^{10}$.

The complex spatiotemporal organization of large-scale neural activity is a robust finding demonstrated across several mammalian species ${ }^{11-16}$ using imaging tools covering an ample range of spatial and temporal scales, including functional magnetic resonance imaging (fMRI) ${ }^{5}$, electroencephalography $(\mathrm{EEG})^{17}$, magnetoencephalography $(\mathrm{MEG})^{18}$, electrocorticography $(\mathrm{ECoG})^{19}$, functional near-infrared spectroscopy

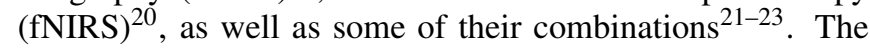
spatial and temporal properties of resting brain activity depend on the global state of consciousness ${ }^{24-27}$, and present alterations specific to several neurological and psychiatric conditions $^{28}$, suggesting their potential application as disease biomarkers in precision medicine ${ }^{29}$. In spite of their obvious relevance for basic and clinical neuroscience, the mechanisms supporting the emergence of complexly patterned spontaneous brain activity remain, to a large extent, unknown.

Computational models show promise to identify some of these mechanisms ${ }^{8}$. Modern neuroimaging techniques are capable of mapping large-scale anatomical and functional con- 
nectivity with unprecedented breadth and accuracy ${ }^{30}$, which can be leveraged to construct whole-brain activity models that reproduce statistical features of empirical data ${ }^{31}$. In these models, local dynamics are coupled by the anatomical connectivity of the brain, and model parameters are then optimized to reproduce certain observables of interest computed from neuroimaging recordings $s^{32}$. By exploring the consequences of changing the local dynamics and global coupling, these semi-empirical ${ }^{33}$ models can inform the mechanisms underlying the features of spontaneous brain activity represented in the chosen observable. Models of whole-brain activity thus constructed have been intensively explored in the past few years to investigate the mechanisms underlying healthy and pathological brain states, to simulate the consequences of externally induced brain stimulation, and with the purpose of data augmentation for machine learning classification of neuroimaging data ${ }^{34-45}$.

The choice of local dynamics determines the range of qualitatively distinct behaviors of the model, its complexity (i.e. number of free parameters), and in turn depends on the desired level of biophysical realism ${ }^{32,46}$. When it comes to modeling the features of spontaneous brain activity recorded with techniques such as fMRI, EEG and MEG, whole-brain models emerge as the natural choice. A common property of phenomenological ${ }^{47}$ models is the inclusion of noise-driven dynamics near a bifurcation to reproduce certain key features of spontaneous brain activity (e.g. metastability, organization into RSN $)^{7,39,48,49}$. For deterministic models attracted to stable or periodic solutions, noise is fundamental to avoid that dynamics become stuck in a state of equilibrium. Thus, the ad hoc introduction of noise in a dynamical system near a bifurcation ensures stochastic switching between different attractors, endowing the simulation with the kind of variability seen in the empirical data.

Another key choice to be made when constructing a wholebrain model is the observable to be reproduced. Since the temporal evolution of complex systems such as the human brain is considered difficult or outright impossible to predict, a meaningful observable should encode the behavior of the system in a statistical sense. Two well-known examples are the functional connectivity (FC) matrix, containing all pairwise correlations between the local activity time series ${ }^{43,50}$, and the functional connectivity dynamics (FCD), which represents the similarity between FC matrices computed over short windows at different times ${ }^{39,49}$. The first of these two observables is useful to represent functional coupling over long or "static" time scales, while the second aims to capture the dynamic switching between metastable states ${ }^{9}$. Other observables can be defined, either assessing static or dynamic characteristics of brain activity.

The possibility of defining multiple meaningful observables raises a problem in the process of constructing a whole-brain activity models based on noise-driven equilibrium dynamics. If a noise-driven system must be poised at a particular combination of parameters (i.e. bifurcation) to ensure the reproduction of a certain statistical observable, does it follow that the same combination of parameters will successfully reproduce other equally meaningful statistical observables? An af- firmative answer seems unlikely. Since a systematic evaluation of how noise-driven equilibrium models perform when simultaneously reproducing multiple observables is lacking, severe limitations could exist for this class of models to explore mechanisms involving more than one feature of spontaneous brain activity. These potential limitations prompt the need to investigate models that are capable of producing complex brain dynamics over a wide range of parameter values.

In this work we explore deterministic chaos as an alternative to noise-induced multistability to reproduce statistical observables computed from resting state fMRI recordings. Chaotic dynamics unfold in the proximity of strange attractors, i.e. complicated fractal sets that give rise to bounded but non-periodic trajectories that are highly dependent on the initial conditions ${ }^{51}$. Among others, mechanisms such as heteroclinic cycling ${ }^{52}$ and chaotic itineracy ${ }^{53}$ endow networked chaotic dynamical systems with complex metastable dynamics in the absence of noise. While the possibility of intrinsically chaotic brain dynamics has been explored in neural recordings acquired from several model organisms investigated at an ample range of spatial and temporal scales ${ }^{54-58}$, the consequences of including deterministic chaos in the local dynamics of semi-empirical whole-brain activity models remains comparatively unexplored. Our purpose is not to engage in the long-standing discussion of deterministic chaos as a fundamental property of brain activity ${ }^{59,60}$. Instead, we are interested in the more pragmatic question of whether deterministic chaos results in models capable of avoiding some limitations intrinsic to noise-driven equilibrium dynamics. In particular, we are interested in how both types of models perform when attempting to simultaneously reproduce multiple empirical observables. Our main motivation is to identify the simplest dynamics capable of reproducing several key features of spontaneous brain activity at the same time, with the purpose of informing the future development of more biophysically realistic models ${ }^{61}$.

For this purpose, we investigated the dynamics of StuartLandau ${ }^{39}$ and Rossler ${ }^{62,63}$ oscillators coupled by an anatomical connectivity network obtained in vivo from diffusion tensor imaging (DTI ${ }^{64}$. These dynamical systems constitute simple examples capable of exhibiting noise-driven multistability and deterministic chaos, respectively. The StuartLandau oscillator undergoes a supercritical Hopf bifurcation, thus capturing the dichotomy between fixed-point noisy dynamics and harmonic oscillatory activity ${ }^{39}$. Due to its conceptual simplicity, it has been used as the basis of phenomenological models in several recent publications $s^{37,38,40,42-44}$. For certain parameters, the Rossler system gives rise to a strange attractor with a single manifold, and is considered one of the simplest examples of chaotic dynamics ${ }^{62}$. We explored and compared how well these two models reproduced several common observables computed from fMRI recordings. 


\section{METHODS}

\section{A. Participants and EEG-fMRI data acquisition}

A cohort of 63 healthy subjects participated in the original experiment (36 females, mean \pm SD age of $23.4 \pm 3.3$ years). Written informed consent was obtained from all subjects. The experimental protocol was approved by the local ethics committee (Goethe-Universität Frankfurt, Germany, protocol number: 305/07). The subjects were reimbursed for their participation. All experiments were conducted in accordance with the relevant guidelines and regulations, and the Declaration of Helsinki.

Participants were scanned for 50 minutes using previously published acquisition parameters ${ }^{26}$. From the group of 63 subjects we selected a subgroup of 9 participants who remained awake throughout the complete duration of the scan (confirmed by assessment of the simultaneous $E E G^{65}$ ). In this way, we obtained unusually long fMRI recordings with the purpose of robustly estimating observables related to FC dynamics.

\section{B. $\quad \mathrm{MRI}$ data preprocessing}

Using Statistical Parametric Mapping (SPM8, www.fil. ion.ucl.ac.uk/spm), raw fMRI data were realigned, normalized and spatially smoothed using a Gaussian kernel with $8 \mathrm{~mm}^{3}$ full width at half maximum. Data was then resampled to $4 \times 4 \times 4 \mathrm{~mm}$ resolution. Note that re-sampling introduced local averaging of blood-oxygen-level-dependent (BOLD) signals, which were eventually averaged over larger cortical and sub-cortical regions of interest as determined by the automatic anatomic labeling (AAL) atlas ${ }^{66}$. Data was denoised by regressing out cardiac, respiratory and residual motion time series ${ }^{67}$, and then band-pass filtered in the $0.01-0.1$ $\mathrm{Hz}$ range using a sixth order Butterworth filter ${ }^{68}$.

\section{Anatomical connectivity matrix}

The anatomical connectivity matrix was obtained applying diffusion tensor imaging (DTI) to diffusion weighted imaging (DWI) recordings from 16 healthy right-handed participants (11 men and 5 women, mean age: $24.75 \pm 2.54$ years) recruited online at Aarhus University, Denmark. Subjects with psychiatric or neurological disorders (or a history thereof) were excluded from participation. We refer to a previous publication for details of the MRI acquisition parameters ${ }^{43}$.

Anatomical connectivity networks were constructed following a three-step process. First, the regions of the wholebrain network were defined using the AAL atlas. Second, the connections between nodes in the whole-brain network (i.e. edges) were estimated applying probabilistic tractography to the DTI data obtained for each participant. Third, results were averaged across participants.

DTI preprocessing was performed using the probtrackx tool of the FSL diffusion imaging toolbox (Fdt) (www.fsl.
fmrib.ox.ac.uk/fsl/fslwiki/FDT) with default parameters. Next, the local probability distributions of fiber directions were estimated at each voxel. The connectivity probability from a seed voxel $i$ to another voxel $j$ was defined as the proportion of fibers passing through voxel $i$ that reached voxel $j$, sampling a total of 5000 streamlines per voxel. This was extended from the voxel to the region level, i.e. in a region of interest consisting of $\mathrm{n}$ voxels, $5000 \times \mathrm{n}$ fibers were sampled. The connectivity probability from region $i$ to region $j$ was calculated as the number of sampled fibers in region $i$ that connected the two regions, divided by $5000 \times \mathrm{n}$, where $\mathrm{n}$ represents the number of voxels in region $i$. The resulting anatomical connectivity matrices were thresholded at $0.1 \%$ (i.e. a minimum of five streamlines), resulting in the anatomical connectivity matrices $C_{i, j}$ used in the models.

\section{Whole-brain model construction}

The general procedure to construct whole-brain models is presented in Fig.1. Following previous work, we constructed computational models of whole-brain activity by assigning local dynamical rules to 90 nodes spanning the whole cortical and subcortical grey matter. These nodes were coupled using an anatomical connectivity matrix $C_{i j}$ which contained in its $i, j$ entry an estimate of the number of white matter tracts connecting nodes $i$ and $j$ (see previous section). We introduced a parameter $G$ to globally scale the $C_{i j}$, thus modeling changes in the overall strength of inter-areal coupling.

To determine the natural frequency of the local dynamics, we estimated the most dominant frequency at each node $\left(\omega_{j}\right)$ as the peak of the Fourier-transformed fMRI time series (averaged across all participants). Consistent with previous reports ${ }^{39,68}$, these frequencies were in the 0.04-0.07 $\mathrm{Hz}$ range.

The fMRI signal corresponding to node $j$ was simulated by the variable $x_{j}$ of the differential equation modeling the local dynamics, integrated using a second-order Runge-Kutta algorithm with a time step of 0.1 . For each model and each parameter combination we produced a number of simulations matching the number of subjects in the dataset. Simulated time series were downsampled to match the sampling frequency of the fMRI data.

\section{Hopf model}

For noise-driven multistability we considered local dynamics given by the Stuart-Landau oscillator, which is equivalent to the normal form of a supercritical Hopf bifurcation. The differential equations for node $j$ are given by,

$$
\begin{aligned}
& \frac{d x_{j}}{d t}=\left[a-x_{j}^{2}-y_{j}^{2}\right] x_{j}-\omega_{j} y_{j}+G \sum_{i} C_{i j}\left(x_{i}-x_{j}\right)+\beta \eta_{j}(t) \\
& \frac{d y_{j}}{d t}=\left[a-x_{j}^{2}-y_{j}^{2}\right] y_{j}+\omega_{j} x_{j}+G \sum_{i} C_{i j}\left(y_{i}-y_{j}\right)+\beta \eta_{j}(t)
\end{aligned}
$$

where $\eta_{j}(t)$ represents additive Gaussian noise, $\beta$ is the noise scaling parameter, $G$ is the anatomical connectivity scaling 


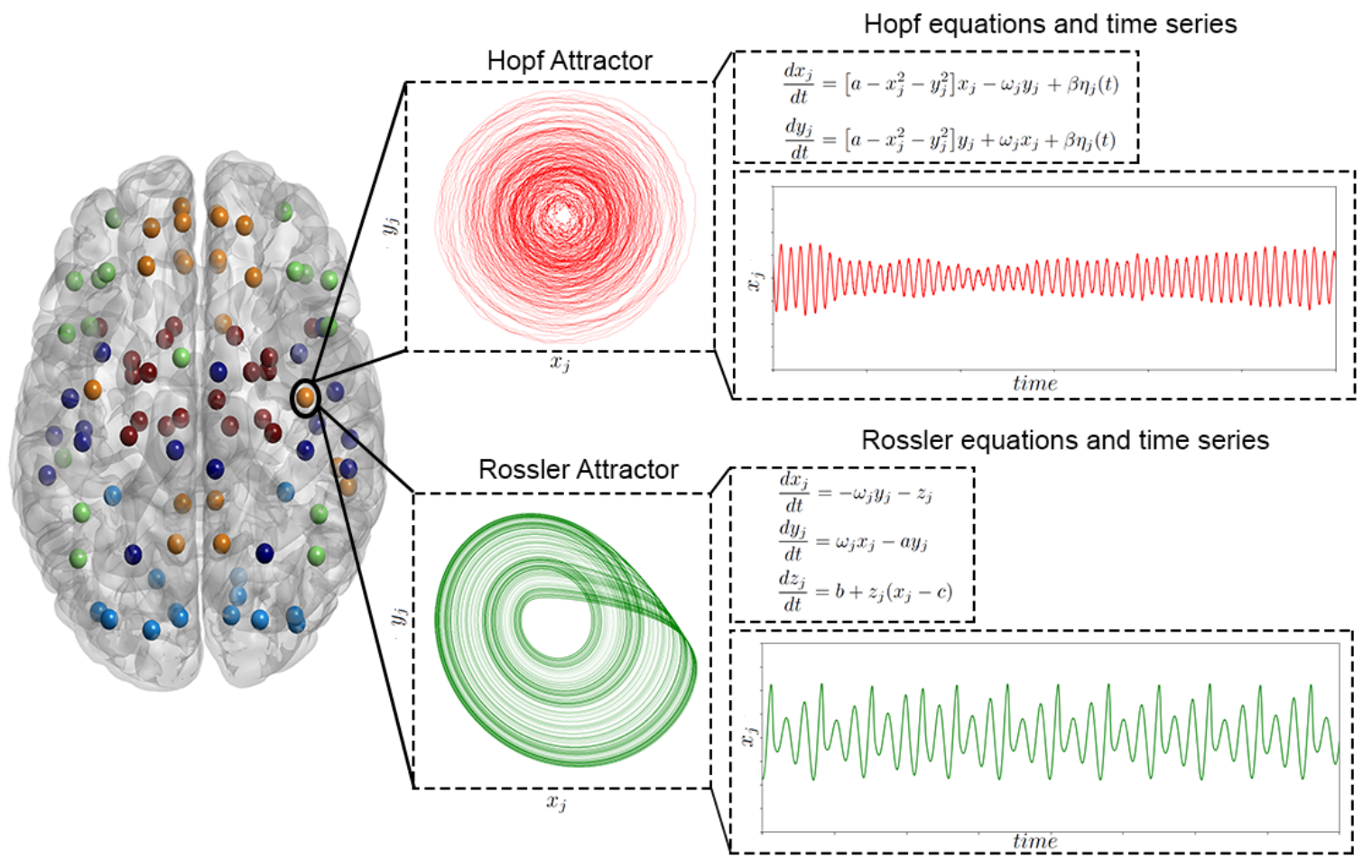

FIG. 1. Schematic of the process followed to construct the whole-brain models. The 90 nodes spanning the whole cortical and subcortical grey matter were coupled by the anatomical connectivity matrix $C_{i, j}$ estimated using probabilistic tractography applied to DTI data. We constructed two models differing in the choice of local dynamics: a model presenting noise-driven multistability (Stuart-Landau oscillators, red), and a model presenting local deterministic chaos (Rossler oscillators, green). In both cases, local dynamics were coupled according to the $C_{i, j}$ matrix scaled by a factor $G$. On the right, we illustrate typical 2D phase space projections and time series for the Stuart-Landau oscillator near the Hopf bifurcation, and for the Rossler oscillator at the chaotic regime. In the first case, dynamics present complex amplitude modulations due to noise-induced switching between the fixed-point and the limit cycle attractors. In the second case dynamics present aperiodic behavior unfolding in the proximity of a strange attractor with a single manifold.

parameter, and $a$ is the bifurcation parameter. For an uncoupled Hopf bifurcation, $a<0$ results in a fixed-point attractor and $a>0$ in an attracting limit cycle, leading to harmonic oscillations at the natural frequency of the node $\omega_{j}$ (estimated from the empirical data). For $a \approx 0$ the system switches between both solutions as a consequence of the additive noise term, as shown in Fig. 1 and Eq. 1 .

\section{Rossler model}

For deterministic chaos we considered local dynamics given by the Rossler oscillator, which is a system of three non-linear ordinary differential equations exhibiting chaotic dynamics for certain parameter combinations. The differential equations for node $j$ are given by,

$$
\begin{aligned}
\frac{d x_{j}}{d t} & =-\omega_{j} y_{j}-z_{j}+G \sum_{i} C_{i j}\left(x_{i}-x_{j}\right) \\
\frac{d y_{j}}{d t} & =\omega_{j} x_{j}-a y_{j} \\
\frac{d z_{j}}{d t} & =b+z_{j}\left(x_{j}-c\right)
\end{aligned}
$$

where $a, b$ and $c$ are free model parameters and $G$ is the global scaling factor of the anatomical connectivity. We fixed parameters $b=0.01$ and $c=13.44$ using a genetic algorithm (see the following section). Since the natural frequencies of brain activity are $\omega_{0} \sim 0.3$, the equations were rescaled using a factor $\gamma=0.3$ to match the empirical frequencies, while preserving the dynamics of the oscillators. We note that the chosen parameters lead to chaotic dynamics (see Figure 1).

\section{E. Rossler parameter selection}

Since there is a mismatch in the number of free parameters between models, we applied a stochastic optimization procedure (genetic algorithm) to fix two of the three parameters of the Rossler model. Following previous work ${ }^{43}$, we used $1 \mathrm{mi}$ nus the structural similarity index (1-SSIM) as the distance metric between simulated and empirical FC matrices ${ }^{69}$. The SSIM is defined as $\left(\frac{2 \mu_{x} \mu_{y}+0.01}{\mu_{x}^{2}+\mu_{y}^{2}+0.01}\right)\left(\frac{2 \sigma_{x} \sigma_{y}+0.03}{\sigma_{x}^{2}+\sigma_{y}^{2}+0.03}\right)\left(\frac{\sigma_{x y}+0.015}{\sigma_{x} \sigma_{y}+0.015}\right)$, where $x$ and $y$ stand for the simulated and average empirical FC, and $\mu_{x}, \mu_{y}, \sigma_{x}, \sigma_{y}$ and $\sigma_{x y}$ correspond to the local means, standard deviations, and co-variances of FC matrices $x$ and $y$, respectively ${ }^{70}$. The SSIM simultaneously factors the corre- 
lation and Frobenius distances between matrices, and can be intuitively understood as an intermediate between both ${ }^{69}$.

The genetic algorithm started with a generation of 10 sets of parameters ("individuals") chosen randomly in the range $[0.0,0.3],[0.0,0.3],[0.0,14]$ and $[0.0,3.0]$ for $a, b, c$ and $G$, respectively. A score proportional to the target function was assigned to each individual. Afterwards, a group of individuals was chosen based on their score ("parents"), and operations of crossover, mutation and elite selection were applied to them to create the next generation. These three operations can be briefly described as follows: 1) elite selection occurs when an individual of a generation shows an extraordinarily low target function in comparison to the other individuals, thus this solution is replicated without changes in the next generation; 2) the crossover operator consists of combining two selected parents to obtain a new individual that carries information from each parent to the next generation; 3) the mutation operator can change one selected individual to induce a random alteration.

Following our previous work, $20 \%$ of each new generation was created by elite selection and $80 \%$ by crossover of the parents, with a $5 \%$ chance of possible mutations within this last group. A new population was thus generated ("offspring") and used iteratively as the next generation until the halting criteria were met, consisting of either reaching an average target function less than $10^{-5}$ across the last 50 generations, or obtaining a constant value for the target function during 50 generations. After applying the optimization algorithm, the parameter values corresponding to the best fit were $a=0.3, b=0.01, c=13.44$ and $G=1.5$. This procedure was used to fix parameters $b$ and $c$. This procedure was used to fix parameters $b$ and $c$. Exploration of parameters $a$ and $G$ was done exhaustively (see section II H).

\section{F. Phase synchrony and metastability.}

We extracted the phases of the band-pass filtered fMRI signals for each of the 90 brain regions and for each subject. The phases were obtained by applying the Hilbert transform to the filtered time series, which resulted in the associated analytic narrowband signal, $a(t)$. The analytic signal $a(t)$ of a signal $x(t)$ is defined as $a(t)=x(t)+i H[x(t)]$, where $i$ is the imaginary unit and $H[x(t)]$ denotes the Hilbert transform of $x(t)$.

We quantified the global degree of synchrony between the nodes across time with the Kuramoto order parameter, $R(t)$, a measure of phase locking given by:

$$
R(t)=\frac{1}{n}\left|\sum_{j} e^{i \phi_{j}}\right|
$$

where $n$ is the total number of nodes and $\phi_{j}(t)$ the instantaneous phase of the narrowband signal at node $j^{44}$. Thus, the Kuramoto order parameter measures the modulus of the average phase of the system at each time point and takes values from 0 to 1 . Here, 0 represents complete absence of phase synchrony and 1 indicates full synchronization.

We calculated the temporal average and standard deviation of the Kuramoto order parameter per subject and subsequently averaged these measures across subjects. We termed these observables synchrony and metastability, respectively. The synchrony represents the global, temporally averaged degree of synchronization between all the nodes in the system, whereas the metastability gives information about temporal variability in the level of synchronization.

\section{G. Quantifying the global Lyapunov exponent}

Establishing whether a system behaves as chaotic depends on the computation of the Lyapunov exponent. In particular, a Maximal Lyapunov exponent (MLE) greater than zero is widely used as an indicator of chaos ${ }^{71}$. To assess the chaotic behavior of the network of coupled Rossler oscillators we calculated the MLE from trajectories in the full variable space, following a standard numerical method published by Sprott $^{72,73}$.

\section{H. Model fitting}

For each distance metric to be optimized, we performed an exhaustive parameter space exploration by varying the two free parameters of the models, corresponding to the global coupling parameter $G$ and the free model parameter $a$, where $a$ was changed homogeneously across all nodes. The global coupling strength $G$ was varied from 0 to 3 in steps of 0.05 , the Hopf bifurcation parameter $a$ from 0.15 to 0.15 (range chosen to include the bifurcation point), and the Rossler parameter $a$ from 0.01 to 0.31 (range chosen to ensure positive Lyapunov exponents of the local dynamics), both in steps of 0.005 . For each parameter combination we computed multiple observables to be defined in the next section; subsequently, we used different distance metrics to compare the empirical and simulated observables. This procedure was performed nine times under exactly the same conditions, and the resulting distance metrics were then averaged, selecting the optimal parameters as those that yielded the lowest value of the averaged distance metric.

\section{Functional connectivity matrix}

We estimated the functional connectivity (FC) matrix for each parameter combination by computing the Pearson correlation coefficient between fMRI signals from all pairs of regions of interest. Subsequently, we calculated the distance between the empirical and simulated FC matrices using three different metrics: Frobenius distance (normalized by the norm of the empirical FC matrix), correlation distance (1-Correlation) and 1-SSIM (defined in a previous section). This resulted in one distance metric value for each parameter combination and per each simulation. 


\section{Comparison between empirical and simulated synchrony and metastability}

The phase synchrony and metastability were computed for each choice of parameters from the filtered simulated time series by applying the same procedure as for the empirical data. We computed the difference between the simulated and empirical observables, and then normalized the results dividing by the empirical value:

$$
M=\frac{O_{\text {simulated }}-O_{\text {empirical }}}{O_{\text {empirical }}}
$$

where $M$ represents the distance metric, $O_{\text {simulated }}$ denotes the synchrony or metastability obtained from the simulated data, and $O_{\text {empirical }}$ denotes the empirical values.

\section{Functional connectivity dynamics}

In order to characterize the time-dependent structure of the resting state fluctuations, we computed the FCD matrices ${ }^{46,49}$. Each full-length BOLD signal of 50 minutes was split up into $M=148$ sliding windows of 60 seconds, overlapping by 40 seconds. For each sliding window centered at time $t$, we calculated an FC matrix, FC $(t)$. The FCD is a $M \times M$ symmetric matrix whose $\left(t_{1}, t_{2}\right)$ entry is defined by the Pearson correlation coefficient between the upper triangular parts of the two matrices $\mathrm{FC}\left(t_{1}\right)$ and $\mathrm{FC}\left(t_{2}\right)$. FCD matrices were computed for each of the nine participants and simulations, also exhaustively exploring the model parameters. In order to capture fluctuations in correlations across the whole signal spectrum, the time series were not filtered when evaluating this observable.

To compare FCD matrices we collected their upper triangular elements (across all participants) and compared the resulting empirical distribution with that obtained across all simulations using the Kolmogorov-Smirnov (KS) statistic as a distance metric. This metric quantifies the maximum difference between the cumulative distribution functions of the two samples.

\section{RESULTS}

\section{A. Group-averaged empirical observables}

We computed a set of statistical observables from empirical resting state fMRI data acquired during long scanning sessions (50 minutes) for 9 healthy awake participants. For this purpose, the blood-oxygen-level-dependent (BOLD) signals corresponding to each voxel were averaged over 90 cortical and sub-cortical regions of interest, determined by the automatic anatomic labeling (AAL) atlas ${ }^{66}$. We obtained the group-level functional connectivity (FC) matrix by computing all pairwise correlations between the BOLD signals corresponding to the 90 regions of interest, averaging afterwards the FC matrices across participants (Fig. 2).
FCD matrices were obtained by computing the correlation between FC matrices corresponding to sliding windows of 60 seconds with a 40 seconds overlap. Fig. 2 displays the FCD matrix and the histogram obtained from the upper-diagonal part of the matrix (pooled across all participants). Other empirical observables related to the dynamics of brain activity, such as phase synchrony and metastability ${ }^{44}$ were computed for each participant and then averaged across the whole sample, following the procedure outlined in the Methods section.
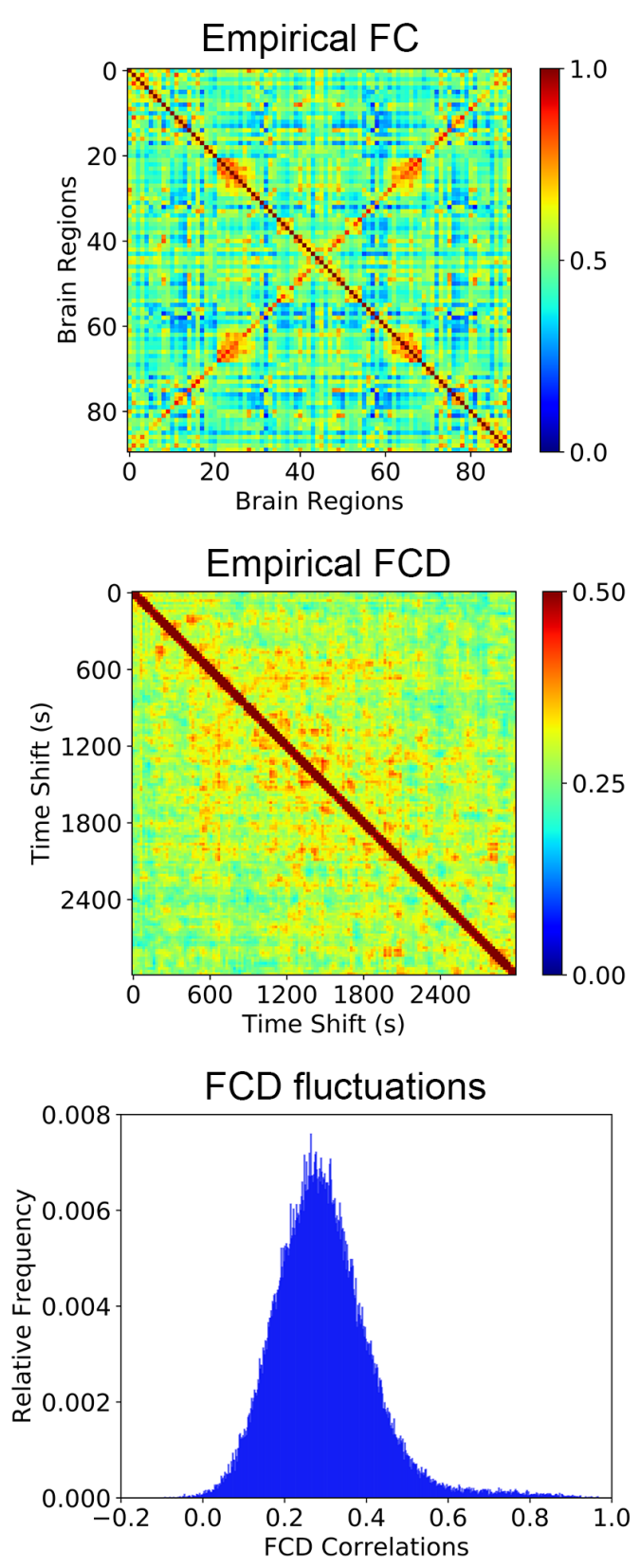

FIG. 2. Average empirical static and dynamic observables. The average FC matrix (top), an example FCD matrix (center), and the histogram of FCD values (bottom) computed from the empirical fMRI data. 


\section{B. Fitting whole-brain models to empirical FC matrices}

We performed an exhaustive exploration of the parameter space for the Hopf and Rossler models, computing each of the three different metrics outlined in the Methods section (1Correlation, 1-SSIM, and Frobenius distance) for each combination of parameter values. The results (averaged across all realizations) are shown in Fig.3A.

As expected from previous work ${ }^{44}$ we observed that for the Hopf model the best fit occurred close to the bifurcation point $(a=0)$ for all metrics. For the Rossler model, instead, comparatively good fits were obtained for the three metrics across a widespread range of parameter values. The global dynamics of the Rossler model presented null and positive Lyapunov exponents across the explored range of parameters (shown as the red curve superimposed over the 1-Correlation panel).

We note that results shown in Fig. 3 were obtained by exploring the Rossler parameter $a$. Figure 1 of the supplementary material contains a reproduction of this figure exploring parameter $b$ instead, showing converging results.

Figure $3 \mathrm{~B}$ shows the FC matrices obtained for the optimal parameter combinations using each metric as target function. We observed higher consistency in the FC matrices simulated with the Rossler model. Further characterization of these results is shown in Fig. 3C, presenting a comparison of the normalized distance ( 1 minus the goodness-of-fit, 1-GoF) of the metrics for the model parameter $a$ optimized according to the Frobenius distance, as a function of the scaling parameter $G$. An optimal coupling parameter could be found for all metrics, with the exception of the Frobenius distance and 1-SSIM in the Hopf model. For the Rossler model, all metrics presented a clearly defined optimal $G$ value, which was also comparatively similar between them.

\section{Fitting whole-brain models to dynamic observables}

Next, we performed the same analysis but with the purpose of reproducing three dynamic observables: the distribution of FCD values, the synchrony, and the metastability. The left panel of Fig. 4A shows the KS distance between the distribution of simulated and empirical FCD values for all parameter combinations. Once more, we can see that the Rossler model presented an widespread region of optimal values. The right panel of the figure shows the histograms of FCD values obtained for the optimal parameters optimized using the KS distance, along with the empirical distribution in blue. We can see that the optimal FCD distribution for the Rossler model appears to be skewed towards the right, while optimal distribution for the Hopf model follows a normal distribution centered around the empirical mean.

Figure 4B shows for both models examples of simulated FCD matrices that minimized the KS distance to the empirical FCD. For the Rossler model, positive correlations were more widespread; in particular, the observation of positive correlations far from the main diagonal implied similar FC matrices for temporal windows at distant times. A similar pattern was observed in the example of the empirical FCD matrix provided in Fig. 2.

We computed the distance between simulated and empirical metastability and synchrony by subtracting the simulated values and normalizing, as explained in the Methods section. Fig. 4C shows how the $\mathrm{KS}$ distance, the metastability $(\mathrm{K})$ and the synchrony $(\mathrm{C})$ distance change as a function of $G$ for the best parameter $a$ obtained using the KS statistic. Neither model presented a minimum of the KS distance curve matching those of the other two metrics.

\section{Comparison of the optimal distance metric values between models}

Figure 5 presents a comparison of $1-\mathrm{GoF}$ for all the metrics and empirical observables reproduced by both models. These values were obtained from the exhaustive optimization procedures presented in Figs. 3 and 4, i.e., we extracted the minimum of each exhaustive exploration plot and condensed all of them in Fig. 5, top panel. We assessed the effect size for the difference between the distributions of optimal distance metrics by means of Cohen's d (Fig. 5, bottom panel) ${ }^{74}$, finding that all distance metrics had similar values for both models, with the exception of the Frobenius distance computed between simulated and empirical FC matrices, for which the Hopf model presented a significantly higher goodness of fit.

\section{E. Fitting multiple simultaneous observables}

We investigated the capacity of each model to simultaneously reproduce multiple observables. For this purpose, we obtained the optimal parameters that reproduced a certain observable (following the exhaustive exploration procedure depicted in Figs. 3 and 4) and then compared how well the dynamics using those parameters could reproduce all other observables.

The results obtained from this analysis are presented in Fig. $6 \mathrm{~A}$. The value in a given matrix entry corresponds to the distance metric indicated in the column evaluated using the parameters that optimize the distance metric indicated in the row. For example, the value 0.28 in the second row and third column of the Hopf model matrix corresponds to the Frobenius distance between the empirical and simulated FC matrices obtained by simulating the Hopf model using the parameters that optimized the 1-SSIM. The bars on the right side of the matrices indicate the sum of values across columns, thus visualizing how well the parameters that optimize the distance metric in each row are capable of reproducing all other observables.

Figure $6 \mathrm{~B}$ presents a graph representation of the generalization matrices in Fig. 6A. Each node represents a distance metric, and an arrow from one node to another indicates that the parameters optimizing the source metric result in low values for the target metric. For a given pair of metrics A and $\mathrm{B}$, we computed the Cohen's d between the optimal distance metric A values obtained after exhaustive exploration of the parameter space, and the distance metric B values obtained 

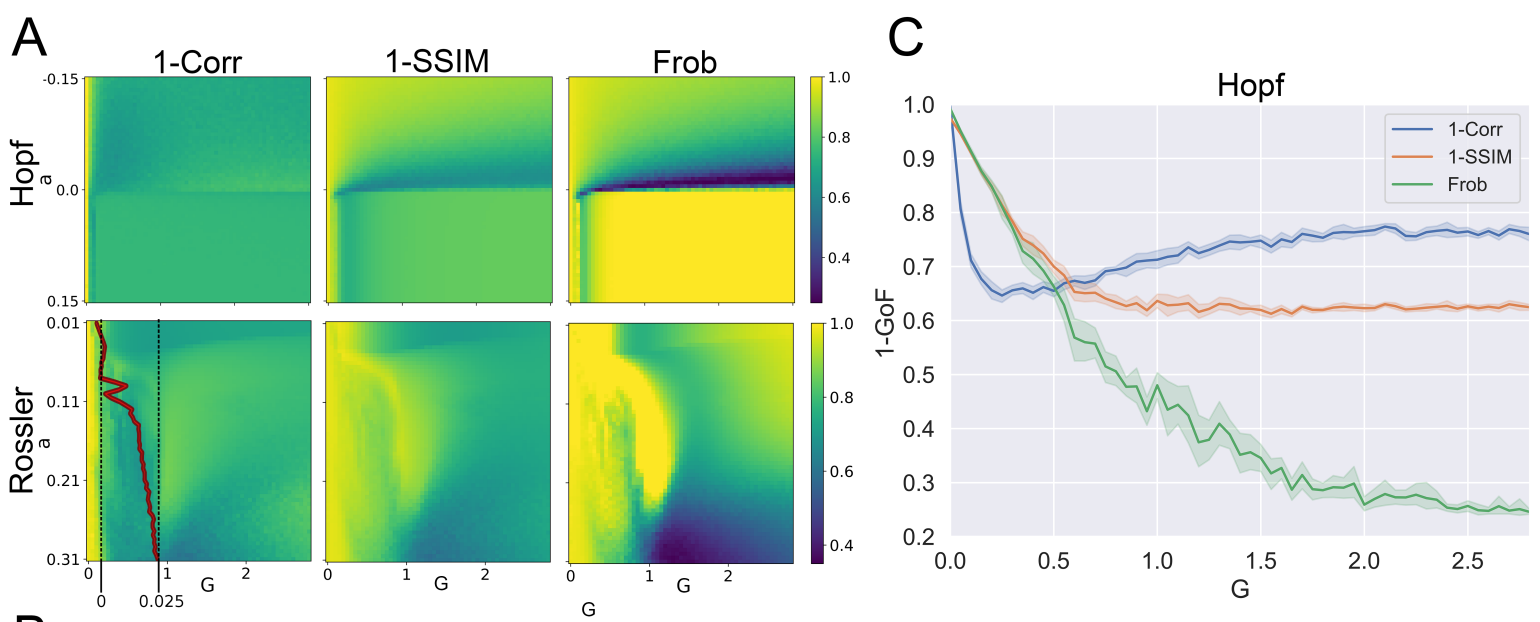

B
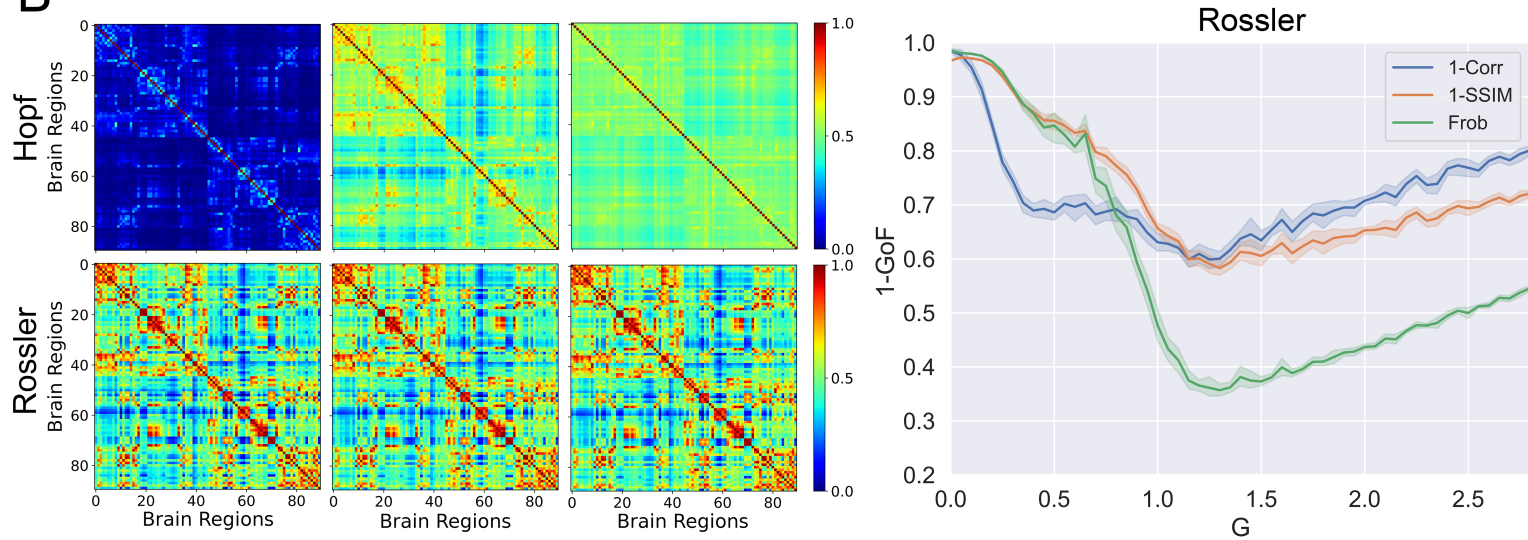

FIG. 3. Whole-brain models fitted to static FC metrics. (a) Exhaustive exploration of the 2D parameter space for the Hopf and Rossler models. The color-coded matrices display the distance between simulated and empirical FC matrices according to three different metrics: 1-Correlation, 1-SSIM, and Frobenius distance. The Lyapunov exponent for the global dynamics of the Rossler model is shown as a red curve superimposed over the 1-Correlation matrix as a function of parameter $a$. (b) The FC matrices computed using the optimal parameter combinations obtained from each distance metric. (c) The three different metrics computed for both models as a function of the coupling parameter $G$, using the best parameter $a$ obtained from optimizing the Frobenius distance.

using the same parameters. A low Cohen's dimplies that both metrics yield similar values for the same model parameters; in other words, that those parameters simultaneously reproduce both observables. We computed the Cohen's d for all pairs of metrics and then applied a threshold to display only the lowest $25 \%$ Cohen's d values as connections between the corresponding nodes.

We can observe that the Hopf model optimized to reproduce $K$ and $C$ performed better than the Rossler model across all other metrics. On the other hand, for FC matrices compared using the 1-Correlation distance, and for FCD distributions compared using the KS distance, the Rossler model outperformed the Hopf model across all other metrics.

An important observation is that, in spite of its comparatively poor performance for $C$ and $K$, the Rossler model optimized to reproduce the empirical dynamics encoded in the FCD matrix resulted in model parameters that also reproduced the "static" FC matrix, according to the three distance criteria (1-Correlation, 1-SSIM and Frobenius distance). Conversely, fitting the Rossler model to the "static" FC resulted in model parameters that approximated the empirical FCD distribution. This behavior was not seen for the Hopf model, for which the optimization of FCD resulted in parameters that failed to approximate the static FC, and vice-versa. In other words, the Rossler model did not force a choice between reproducing the FC over long temporal scales (FC matrix) and reproducing the FCD.

\section{F. Comparison of generalization performance between models fitted to FC and FCD}

Figure 7 illustrates how the Rossler model is capable of simultaneously approximating static and dynamic FC, while the Hopf model produces inconsistent results when optimized to reproduce one of them and is then tested to reproduce the other. The first row of both left and right panels shows the optimal FC matrix (using the Frobenius distance) and the dis- 

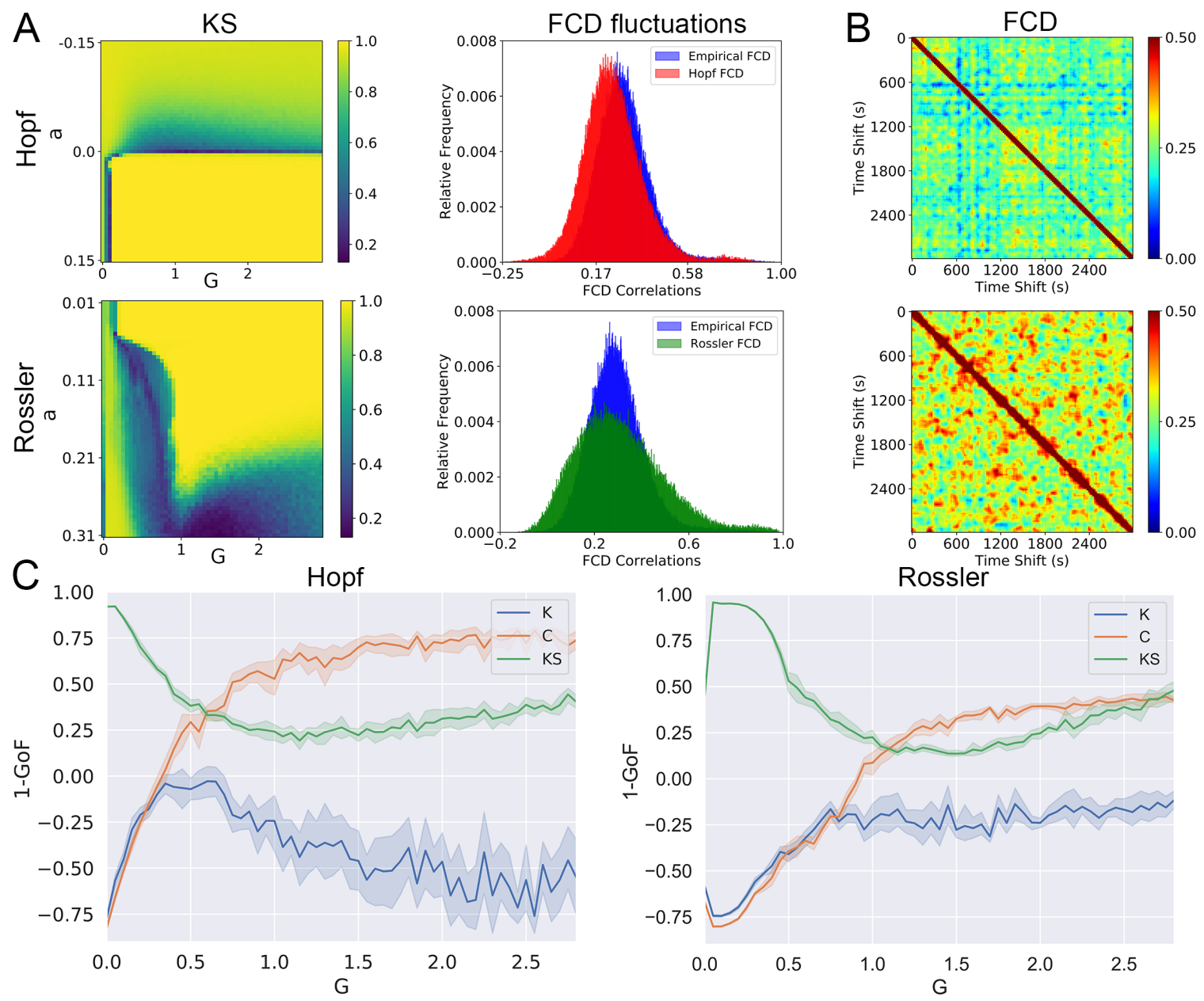

FIG. 4. Whole-brain models fitted to dynamic observables. (a) KS distance between the distribution of simulated and empirical FCD values computed for all parameter combinations and for both models. Note that the best fit was obtained for $a \approx 0$ for the Hopf model and for high $a$ values for the Rossler model. The right panel displays the optimal distribution of FCD values for both models, together with the empirical distribution. (b) Examples of FCD matrices for both models computed using the optimal parameters obtained from the optimization of the KS distance. (c) KS distance, metastability (K) and synchrony (C) distance between simulated and empirical data as a function of $G$, computed for the best $a$ obtained using the KS statistic.

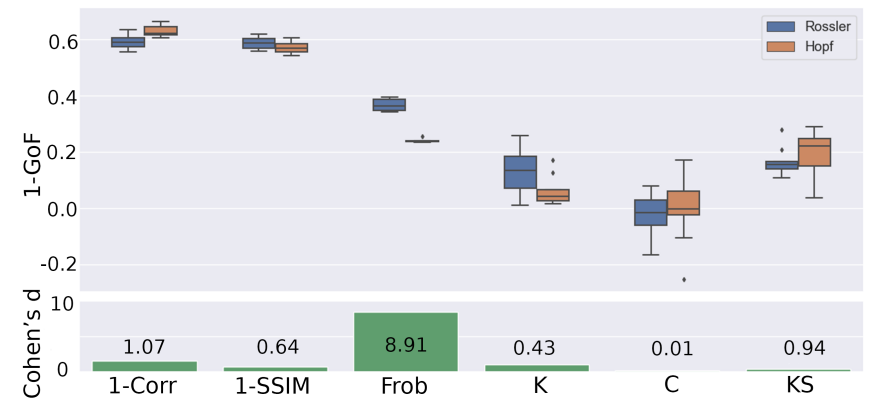

FIG. 5. Comparison of 1 minus the goodness-of-fit $(1-G o F)$ for all metrics and observables between both models. The bottom panel presents the effect size for the difference in terms of Cohen's d.

tribution of FCD values for the parameters that optimize the reproduction of the FC matrix. Conversely, the second row shows the FC matrix computed using the parameters that minimize the KS distance between empirical and FCD distributions, and then the distribution of FCD values using those parameters.

From the left panel, it is clear that the Hopf model tuned to reproduce the FC matrix grossly misrepresented the distribution of FCD values and vice-versa. As expected from the results presented in Fig. 6, the Rossler model simultaneously approximated the FC matrix and the distribution of FCD values with the same combination of parameters.

\section{DISCUSSION}

Speaking of Newton's second law, the British astronomer Arthur Eddington once said: "A force is whatever we need to put on left side of the equation to obtain results that agree with the observed motions"75. In this view, physics is interpreted as 

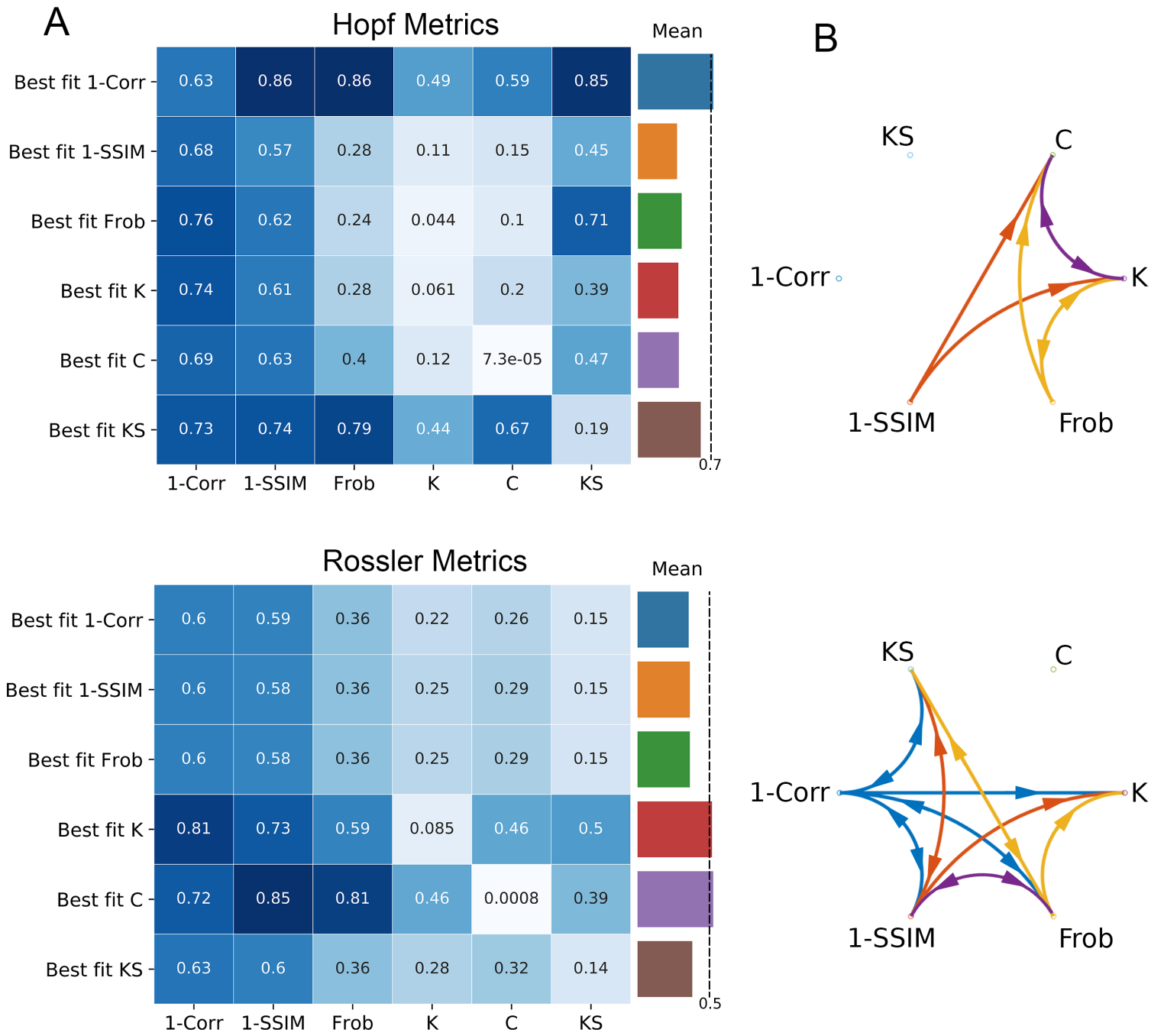

FIG. 6. Generalization between empirical observables. (a) Matrix containing the distance metrics indicated in the columns evaluated using the parameters that optimize the distance metrics indicated in the rows. The bars on the right side of the matrix indicate the average across all columns. (b) Each distance metric is represented as a node in a graph where arrows indicate that the parameters optimizing the source metric result in low values for the target metric as well. For metrics A and B (represented by two nodes in the graph), we computed the Cohen's d between the optimal distance metric A values obtained after exhaustive exploration of the parameter space, and the distance metric B values obtained using those same parameters. We applied a threshold to keep $25 \%$ of the connections with the lowest Cohen's d.

an effort to produce increasingly more accurate models capable of describing the dynamics found in nature. Contemporary physics has become entangled with abstract mathematics to the point that formal structures are by themselves considered an important guide for theory building. In comparison, theoretical neuroscience is a much younger field still in need of exploring different mechanisms to explain the dynamics observed in experimental data ${ }^{61}$, a project having much in common with Eddington's interpretation of Newton's second law.

Ever since the discoveries of Hodgkin and Huxley, computational models of neural activity can be formulated with a high degree of biophysical realism ${ }^{76}$. Advances in microscopy and related technologies have contributed to unveil intricate networks of synaptic connections at the scale of single neurons, allowing to reconstruct the wiring of com- plete cortical columns ${ }^{77}$. Large collaborative efforts, such as the Human Brain Project, strive to combine this information to produce highly detailed simulations of small cortical regions ${ }^{78}$. As computational power is increased and the available maps of synaptic connectivity are expanded, it is expected that realistic models based on multi-objective optimization over a large parameter space will begin to furnish predictions at the macroscopic scale, i.e. the scale investigated with neuroimaging tools such as fMRI, EEG and MEG. These predictions will have to be consistent with the phenomenological models that successfully described the dynamics and functional connectivity of large-scale brain activity. Thus, our investigation of the potential mechanisms underlying dynamics at this scale is motivated by the need to inform and constraint the development of more biophysically realistic mod- 

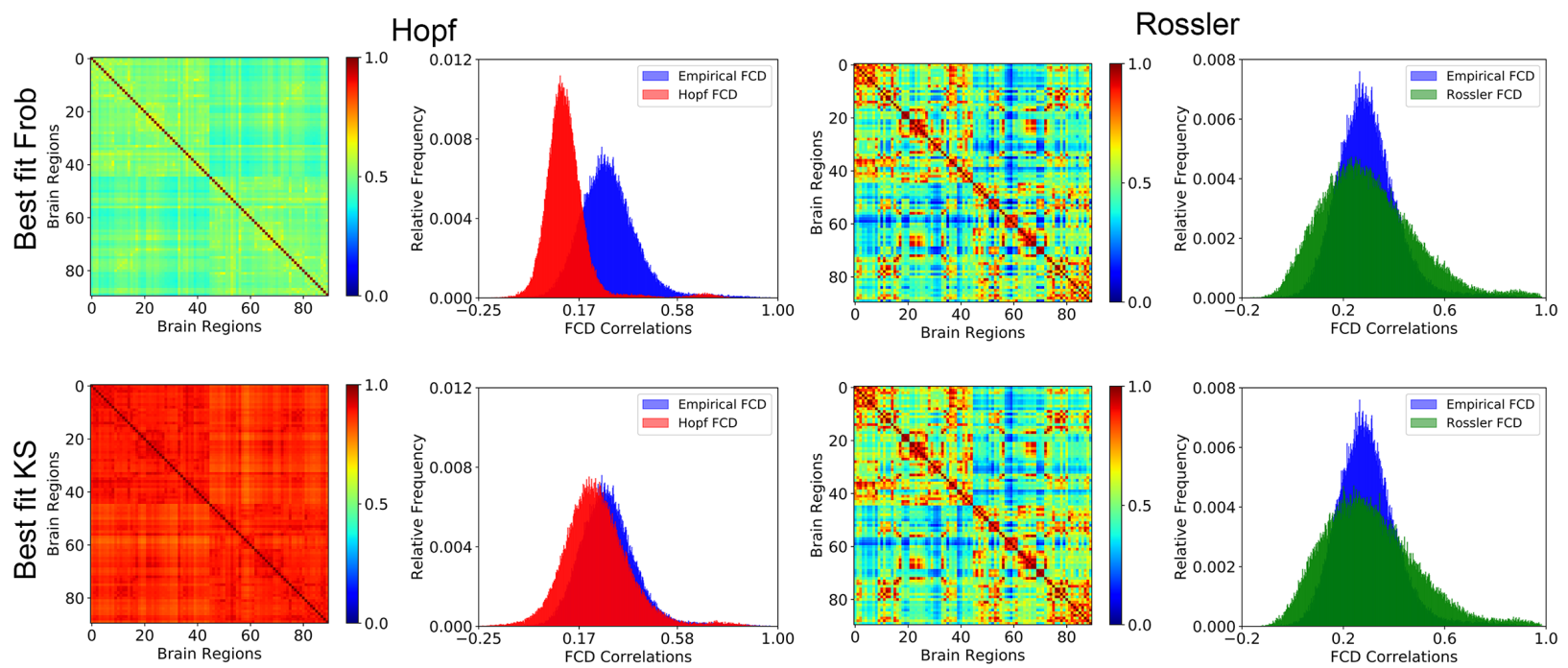

FIG. 7. The Rossler model is capable of simultaneously approximating the empirical FC matrix and the distribution of FCD values. Each panel (left and right) contains in its first row the best reproduction of the FC matrix (using the Frobenius distance) and the distribution of FCD values for the parameters that optimize the reproduction of the FC matrix; conversely, the second row shows the FC matrix computed using the parameters that minimize the KS distance between empirical and FCD distributions, and then the distribution of FCD values using those parameters. The left panel illustrates the failure of the Hopf model to simultaneously reproduce both observables, which is achieved by the Rossler model, as shown in the right panel.

els.

We explored two different mechanisms behind the complex spatiotemporal dynamics of resting state brain activity. As shown in Figs. 3 and 4, a model based on noise-driven multistability between equilibrium solutions (Hopf model) required fine-tuning of the bifurcation parameter to adequately reproduce different empirical observables. Conversely, a model based on deterministic chaos (Rossler model) reproduced these observables over a range of the model parameter, for which the global dynamics also exhibited positive Lyapunov exponents. However, it must be noted that due to the different nature of both models, the parameters do not play the same role in determining the dynamics of the systems, and hence they are not directly comparable. This is limitation is inherent to the comparison between models presenting qualitatively different dynamics.

Computational models are developed and implemented with the purpose of capturing certain features of brain activity, which depend on the scientific question and its associated hypotheses. However, models can be difficult to interpret if they are inconsistent when attempting to reproduce multiple features at the same time. As shown in the left panel of Fig. 7, the optimal fit of the Hopf model to the FCD distribution results in parameters that produce a meaningless FC matrix, which is perhaps the most widespread summary statistic computed from resting state fMRI data ${ }^{4}$. The failure of noise-driven equilibrium models to reproduce multiple observables at the same time is also highlighted by the recent work of Courtiol and colleagues ${ }^{79}$, who performed exhaustive explorations of the parameter space to reveal the presence of very different optimal working points in systems of coupled oscillators. Ideally, phenomenological models of whole-brain activity should be capable of approximating both the "static" and dynamic FC; however, this could be difficult for noise-driven equilibrium models which require fine-tuning to dynamical criticality to produce rich temporal FC fluctuations. In the Rossler model we found a widespread range of model parameters that simultaneously fitted multiple empirical observables. This result suggests that the dichotomy between reproducing static or dynamic FC features could be avoided by the introduction of deterministic chaos in the local dynamics. Another possibility to avoid this issue is to construct models to directly reproduce the temporal evolution of neural activity time series; however, these models require as an input some type of empirical signal adequately sampled in the temporal domain ${ }^{80}$.

Models based on noise-driven multistability have found widespread applications in computational neuroscience ${ }^{81}$. Since their dynamics can be understood in terms of attractors connected by noise-induced transitions, these models are easier to interpret and construct with the purpose of producing certain predefined behaviors. Within the specific context of whole-brain models, the interplay between noise and deterministic equilibrium dynamics is sufficient to generate ongoing dynamics capable of exploring the repertoire of potential brain configurations ${ }^{7}$. The noise-driven exploration of this repertoire produces the dynamic fluctuations in functional connectivity that have been robustly established using several imaging modalities ${ }^{23,49,82}$. In general, the qualitative global behavior of whole-brain models can be understood in terms of the bifurcation diagrams of the local dynamics, at least in the case of weak coupling. Conversely, while coupled chaotic systems can exhibit multistable swiching by mechanisms such as chaotic itineracy ${ }^{53}$ and heteroclinic cycling ${ }^{52}$, local dynamics cannot be easily understood by bifurcation analysis due to 
the presence of strange attractors.

The inclusion of noise should not be disregarded as an ad hoc mechanism required to produce interesting dynamics. Instead, it should be considered as the potential manifestation of biological and physical processes occurring at multiple spatial and temporal scales. Neural systems are subject to a number of noise sources: the activity of a population of neurons embodied in the brain inevitably occurs in the presence of stochastic fluctuations due to thermal energy, ion channel chattering, intermittent neurotransmitter release, and irregular synaptic inputs from other neurons, among other sources of noise ${ }^{81,83}$. However, the biological interpretation of additive noise terms in large-scale models of whole-brain activity remains unclear. Following the principle of economy of explanation ${ }^{84}$, unknown sources of variability should be considered less satisfactory than endogenous variability produced by intrinsically non-equilibrium dynamics. Even though deterministic chaos may be appealing for large-scale phenomenological models, it is less clear how to systematically construct biophysically realistic models whose variability stems from chaotic dynamics, and whose output is consistent with experimental data.

Another relevant point is whether empirical data supports the presence of chaos in brain dynamics. This is a contentious issue, possibly because current experimental tools are insufficient to produce evidence that can be considered as definitive. Over the past decades, several studies reported the presence of chaotic dynamics in time series obtained from an ample variety of neural systems ${ }^{59,60}$; however, an equally large literature has been published arguing against this possibility ${ }^{85,86}$. Theoretically, it is accepted that the inherent instability of chaotic dynamics facilitates the extraordinary ability of neural systems to respond quickly to changes in their external inputs ${ }^{87}$, to flexibly transition between behavioral patterns as a consequence of environmental changes, and to explore the large repertoire of dynamical states that endows neural circuits with remarkable computational capabilities ${ }^{88}$. Chaotic dynamics in the brain could emerge in several ways, such as from intrinsic mechanisms within individual neurons ${ }^{89}$, or from the collective dynamics of neural networks ${ }^{90-92}$. While our results do not demonstrate the presence of deterministic chaos in large-scale brain activity, they illustrate how even the simplest model of coupled chaotic oscillators is capable simultaneously reproducing "static" and dynamic FC observables. This result should be taken into consideration by future model building efforts, independently of the deeper question of whether chaotic dynamics represents an intrinsic feature of brain activity.

It must be stressed that noise and deterministic chaos are not mutually exclusive mechanisms to produce multistable brain dynamic. As recently shown by Orio et al. ${ }^{93}$, moderate noise can enhance the multistable behavior of chaotic neural networks, resulting in an ampler exploration of the synchronization repertoire, with very high levels of noise eventually abolishing multistability. While this study investigated a conductance-based neural model wired with a smallworld network topology, presumably noise-enhanced multistable chaotic dynamics can also be found in Rossler oscilla- tors coupled by realistic anatomical connectivity, a possibility that should be addressed by future studies.

Our study presents some limitations pointing the way towards future improvements. First, while we considered instantaneous interactions between the network nodes, the interplay between noise and conduction delay is a key factor to reproduce the dynamics of spontaneous brain activity fluctuations ${ }^{94}$. However, the combination of slow temporal sampling by fMRI and fast conduction speeds through long-range myelinated axons likely attenuates the effects of omitting delays in our models. As shown by Petkoski et al. $(2018)^{95}$ and Petkoski and Jirsa (2019) ${ }^{63}$, transmission delays can impact on the frequency-specific dynamics of simulated brain activity; also, as shown by Cabral et al. $(2014)^{96}$, the inclusion of transmission delays is important to reproduce empirical correlations between narrow band MEG frequency envelopes. However, this information is not obtainable from fMRI recordings and thus cannot be reproduced by our models. Second, we did not obtain the anatomical connectivity networks from the same subjects who were recorded with fMRI. Instead, DTI recordings were obtained from another group of healthy participants who can be considered as representative of an adult population. However, individual connectivity matrices should be used when modeling the large-scale dynamics of individuals who could present structural abnormalities as a consequence of neurological or psychiatric impairments. Third, the output of our model was interpreted as the fMRI signals, from which we constructed the simulated observables. This approach neglects the possibility of multiscale interactions shaping the results; i.e. the modeling of faster time-scales which, in turn, determine the slower BOLD fluctuations ${ }^{80,96}$. Future studies based on empirical data with better temporal resolution (e.g. MEG) should explore this possibility in the context of chaotic vs. stochastic dynamics. Finally, we focused on modeling unusually long recordings (50 minutes) of awake subjects, which contributed towards more robust estimates of dynamic FC measures. However, the biological implications of our findings should be explored by investigating subjects during other states of consciousness, e.g. states of such as deep sleep, general anaesthesia, or in patients diagnosed with disorders of consciousness ${ }^{97}$. It is tempting to speculate that the level of consciousness will be paralleled by the degree of chaoticity in the best fitting model, a possibility that will be explored in future studies.

In conclusion, we showed that chaotic dynamics give rise to some interesting features in whole-brain activity models, outperforming noise-driven equilibrium models in the simultaneous reproduction of multiple empirical observables. While simplified phenomenological models may appear to be overly detached from the intricate details of the human brain, they are nevertheless important to propose conceptually simple mechanisms that more realistic models should eventually strive to reproduce. Our results identified some attractive features of deterministic chaos that should not be neglected by future modeling efforts, even in the simultaneous presence of noisy inputs. Facing up to the challenge of imprinting and interpreting deterministic chaos in more realistic whole-brain models could be a key to understand how the human brain is capa- 
ble of producing an ever-changing stream of complex activity patterns.

\section{SUPPLEMENTARY MATERIAL}

The supplementary material contains a reproduction of Fig. 3 exploring other parameters of the Rossler system and a figure displaying the Cohen's $d$ values of the arrows drawn in Fig. 6B.

\section{ACKNOWLEDGMENTS}

This work was supported by funding from Agencia Nacional De Promocion Cientifica Y Tecnologica (Argentina), grant PICT-2018-03103. The authors acknowledge the Toyoko 2020 program for granting cloud computing services.

\section{DATA AVAILABILITY}

The data that support the findings of this study are openly available at ${ }^{98}$

${ }^{1}$ Here we employ the term large-scale as synonym of brain-wide, in reference to macroscopic brain activity spanning from few centimeters up to the whole cortex.

${ }^{2}$ M. E. Raichle, "The brain's dark energy," Science-New York Then Washington- 314, 1249 (2006).

${ }^{3}$ G. Deco, V. K. Jirsa, and A. R. McIntosh, "Emerging concepts for the dynamical organization of resting-state activity in the brain," Nature Reviews Neuroscience 12, 43-56 (2011).

${ }^{4}$ M. P. Van Den Heuvel and H. E. H. Pol, "Exploring the brain network: a review on resting-state fmri functional connectivity," European neuropsychopharmacology 20, 519-534 (2010).

${ }^{5}$ J. S. Damoiseaux, S. Rombouts, F. Barkhof, P. Scheltens, C. J. Stam, S. M. Smith, and C. F. Beckmann, "Consistent resting-state networks across healthy subjects," Proceedings of the national academy of sciences 103, 13848-13853 (2006).

${ }^{6}$ S. M. Smith, P. T. Fox, K. L. Miller, D. C. Glahn, P. M. Fox, C. E. Mackay, N. Filippini, K. E. Watkins, R. Toro, A. R. Laird, et al., "Correspondence of the brain's functional architecture during activation and rest," Proceedings of the national academy of sciences 106, 13040-13045 (2009).

${ }^{7}$ G. Deco and V. K. Jirsa, "Ongoing cortical activity at rest: criticality, multistability, and ghost attractors," Journal of Neuroscience 32, 3366-3375 (2012).

${ }^{8}$ G. Deco, V. K. Jirsa, and A. R. McIntosh, "Resting brains never rest: computational insights into potential cognitive architectures," Trends in neurosciences 36, 268-274 (2013).

${ }^{9}$ F. Cavanna, M. G. Vilas, M. Palmucci, and E. Tagliazucchi, "Dynamic functional connectivity and brain metastability during altered states of consciousness," Neuroimage 180, 383-395 (2018).

${ }^{10}$ M. W. Cole, T. Ito, D. S. Bassett, and D. H. Schultz, "Activity flow over resting-state networks shapes cognitive task activations," Nature neuroscience 19, 1718-1726 (2016).

${ }^{11}$ R. M. Hutchison, L. S. Leung, S. M. Mirsattari, J. S. Gati, R. S. Menon, and S. Everling, "Resting-state networks in the macaque at $7 \mathrm{t}$," Neuroimage 56, 1546-1555 (2011).

${ }^{12}$ C. P. Pawela, B. B. Biswal, Y. R. Cho, D. S. Kao, R. Li, S. R. Jones, M. L. Schulte, H. S. Matloub, A. G. Hudetz, and J. S. Hyde, "Resting-state functional connectivity of the rat brain," Magnetic Resonance in Medicine: An Official Journal of the International Society for Magnetic Resonance in Medicine 59, 1021-1029 (2008).
${ }^{13}$ D. Szabo, K. Czeibert, Á. Kettinger, M. Gácsi, A. Andics, Á. Miklósi, and E. Kubinyi, "Resting-state fmri data of awake dogs (canis familiaris) via group-level independent component analysis reveal multiple, spatially distributed resting-state networks," Scientific reports 9, 1-25 (2019).

${ }^{14}$ Z. C. Zhou, A. P. Salzwedel, S. Radtke-Schuller, Y. Li, K. K. Sellers, J. H. Gilmore, Y.-Y. I. Shih, F. Fröhlich, and W. Gao, "Resting state network topology of the ferret brain," Neuroimage 143, 70-81 (2016).

${ }^{15}$ A. M. Belcher, C. C. Yen, H. Stepp, H. Gu, H. Lu, Y. Yang, A. C. Silva, and E. A. Stein, "Large-scale brain networks in the awake, truly resting marmoset monkey," Journal of neuroscience 33, 16796-16804 (2013).

${ }^{16}$ F. Sforazzini, A. J. Schwarz, A. Galbusera, A. Bifone, and A. Gozzi, "Distributed bold and cbv-weighted resting-state networks in the mouse brain," Neuroimage 87, 403-415 (2014).

${ }^{17}$ A. Custo, D. Van De Ville, W. M. Wells, M. I. Tomescu, D. Brunet, and C. M. Michel, "Electroencephalographic resting-state networks: source localization of microstates," Brain connectivity 7, 671-682 (2017).

${ }^{18}$ M. J. Brookes, M. Woolrich, H. Luckhoo, D. Price, J. R. Hale, M. C. Stephenson, G. R. Barnes, S. M. Smith, and P. G. Morris, "Investigating the electrophysiological basis of resting state networks using magnetoencephalography," Proceedings of the National Academy of Sciences 108, 16783-16788 (2011).

${ }^{19}$ C. D. Hacker, A. Z. Snyder, M. Pahwa, M. Corbetta, and E. C. Leuthardt, "Frequency-specific electrophysiologic correlates of resting state fmri networks," Neuroimage 149, 446-457 (2017).

${ }^{20}$ L. Duan, Y.-J. Zhang, and C.-Z. Zhu, "Quantitative comparison of restingstate functional connectivity derived from fnirs and fmri: a simultaneous recording study," Neuroimage 60, 2008-2018 (2012).

${ }^{21}$ H. Laufs, K. Krakow, P. Sterzer, E. Eger, A. Beyerle, A. Salek-Haddadi, and A. Kleinschmidt, "Electroencephalographic signatures of attentional and cognitive default modes in spontaneous brain activity fluctuations at rest," Proceedings of the national academy of sciences 100, 11053-11058 (2003).

${ }^{22}$ D. Mantini, M. G. Perrucci, C. Del Gratta, G. L. Romani, and M. Corbetta, "Electrophysiological signatures of resting state networks in the human brain," Proceedings of the National Academy of Sciences 104, 13170 13175 (2007).

${ }^{23}$ E. Tagliazucchi, F. Von Wegner, A. Morzelewski, V. Brodbeck, and H. Laufs, "Dynamic bold functional connectivity in humans and its electrophysiological correlates," Frontiers in human neuroscience 6, 339 (2012).

${ }^{24}$ A. Demertzi, E. Tagliazucchi, S. Dehaene, G. Deco, P. Barttfeld, F. Raimondo, C. Martial, D. Fernández-Espejo, B. Rohaut, H. Voss, et al., "Human consciousness is supported by dynamic complex patterns of brain signal coordination," Science advances 5, eaat7603 (2019).

${ }^{25}$ E. Tagliazucchi, F. von Wegner, A. Morzelewski, V. Brodbeck, K. Jahnke, and H. Laufs, "Breakdown of long-range temporal dependence in default mode and attention networks during deep sleep," Proceedings of the National Academy of Sciences 110, 15419-15424 (2013).

${ }^{26} \mathrm{E}$. Tagliazucchi and H. Laufs, "Decoding wakefulness levels from typical fmri resting-state data reveals reliable drifts between wakefulness and sleep," Neuron 82, 695-708 (2014).

${ }^{27}$ P. Barttfeld, L. Uhrig, J. D. Sitt, M. Sigman, B. Jarraya, and S. Dehaene, "Signature of consciousness in the dynamics of resting-state brain activity," Proceedings of the National Academy of Sciences 112, 887-892 (2015).

${ }^{28} \mathrm{M}$. Greicius, "Resting-state functional connectivity in neuropsychiatric disorders," Current opinion in neurology 21, 424-430 (2008).

${ }^{29}$ A. T. Drysdale, L. Grosenick, J. Downar, K. Dunlop, F. Mansouri, Y. Meng, R. N. Fetcho, B. Zebley, D. J. Oathes, A. Etkin, et al., "Resting-state connectivity biomarkers define neurophysiological subtypes of depression," Nature medicine 23, 28-38 (2017).

${ }^{30}$ M. F. Glasser, S. M. Smith, D. S. Marcus, J. L. Andersson, E. J. Auerbach, T. E. Behrens, T. S. Coalson, M. P. Harms, M. Jenkinson, S. Moeller, et al., "The human connectome project's neuroimaging approach," Nature neuroscience 19, 1175-1187 (2016).

${ }^{31}$ P. Sanz-Leon, S. A. Knock, A. Spiegler, and V. K. Jirsa, "Mathematical framework for large-scale brain network modeling in the virtual brain," Neuroimage 111, 385-430 (2015).

${ }^{32}$ M. Breakspear, "Dynamic models of large-scale brain activity," Nature neuroscience 20, 340-352 (2017).

${ }^{33}$ A semi-empirical model combines simulated local dynamics with empirical estimates of anatomical and functional connectivity. 
${ }^{34}$ G. Deco, J. Cruzat, J. Cabral, G. M. Knudsen, R. L. Carhart-Harris, P. C. Whybrow, N. K. Logothetis, and M. L. Kringelbach, "Whole-brain multimodal neuroimaging model using serotonin receptor maps explains nonlinear functional effects of 1sd," Current biology 28, 3065-3074 (2018).

${ }^{35}$ V. K. Jirsa, T. Proix, D. Perdikis, M. M. Woodman, H. Wang, J. GonzalezMartinez, C. Bernard, C. Bénar, M. Guye, P. Chauvel, et al., "The virtual epileptic patient: individualized whole-brain models of epilepsy spread," Neuroimage 145, 377-388 (2017).

${ }^{36}$ M. L. Kringelbach, J. Cruzat, J. Cabral, G. M. Knudsen, R. Carhart-Harris, P. C. Whybrow, N. K. Logothetis, and G. Deco, "Dynamic coupling of whole-brain neuronal and neurotransmitter systems," Proceedings of the National Academy of Sciences 117, 9566-9576 (2020).

${ }^{37}$ G. Deco, J. Cruzat, J. Cabral, E. Tagliazucchi, H. Laufs, N. K. Logothetis, and M. L. Kringelbach, "Awakening: Predicting external stimulation to force transitions between different brain states," Proceedings of the National Academy of Sciences 116, 18088-18097 (2019).

${ }^{38}$ G. Deco, J. Cabral, V. M. Saenger, M. Boly, E. Tagliazucchi, H. Laufs, E. Van Someren, B. Jobst, A. Stevner, and M. L. Kringelbach, "Perturbation of whole-brain dynamics in silico reveals mechanistic differences between brain states," Neuroimage 169, 46-56 (2018).

${ }^{39}$ G. Deco, M. L. Kringelbach, V. K. Jirsa, and P. Ritter, "The dynamics of resting fluctuations in the brain: metastability and its dynamical cortical core," Scientific reports 7, 1-14 (2017).

${ }^{40}$ Y. S. Perl, C. Pallacivini, I. P. Ipina, M. L. Kringelbach, G. Deco, H. Laufs, and E. Tagliazucchi, "Data augmentation based on dynamical systems for the classification of brain states," bioRxiv (2020).

${ }^{41}$ Y. S. Perl, H. Boccacio, I. Pérez-Ipiña, F. Zamberlán, H. Laufs, M. Kringelbach, G. Deco, and E. Tagliazucchi, "Generative embeddings of brain collective dynamics using variational autoencoders," arXiv preprint arXiv:2007.01378 (2020).

${ }^{42}$ Y. S. Perl, C. Pallavicini, I. P. Ipina, A. Demertzi, V. Bonhomme, C. Martial, R. Panda, J. Annen, A. Ibanez, M. Kringelbach, et al., "Perturbations in dynamical models of whole-brain activity dissociate between the level and stability of consciousness," bioRxiv (2020).

${ }^{43}$ I. P. Ipiña, P. D. Kehoe, M. Kringelbach, H. Laufs, A. Ibañez, G. Deco, Y. S. Perl, and E. Tagliazucchi, "Modeling regional changes in dynamic stability during sleep and wakefulness," NeuroImage , 116833 (2020).

${ }^{44}$ B. M. Jobst, R. Hindriks, H. Laufs, E. Tagliazucchi, G. Hahn, A. PonceAlvarez, A. B. Stevner, M. L. Kringelbach, and G. Deco, "Increased stability and breakdown of brain effective connectivity during slow-wave sleep: mechanistic insights from whole-brain computational modelling," Scientific reports 7, 1-16 (2017).

${ }^{45}$ T. Kunze, A. Hunold, J. Haueisen, V. Jirsa, and A. Spiegler, "Transcranial direct current stimulation changes resting state functional connectivity: A large-scale brain network modeling study," Neuroimage 140, 174-187 (2016).

${ }^{46}$ G. Deco, V. K. Jirsa, P. A. Robinson, M. Breakspear, and K. Friston, "The dynamic brain: from spiking neurons to neural masses and cortical fields," PLoS Comput Biol 4, e1000092 (2008).

${ }^{47}$ Phenomenological models are concerned with simple mechanisms that produce dynamics matching those observed in the experimental data. The differential equations and their parameters are not chosen to be biophysically realistic, but instead to display certain desired qualitative behaviors seen in the data. However, the dynamics of realistic models can be locally reduced to phenomenological models by finding their normal form.

${ }^{48}$ M. Golos, V. Jirsa, and E. Daucé, "Multistability in large scale models of brain activity," PLoS computational biology 11, e1004644 (2015).

${ }^{49}$ E. C. Hansen, D. Battaglia, A. Spiegler, G. Deco, and V. K. Jirsa, "Functional connectivity dynamics: modeling the switching behavior of the resting state," Neuroimage 105, 525-535 (2015).

${ }^{50}$ S. M. Smith, D. Vidaurre, C. F. Beckmann, M. F. Glasser, M. Jenkinson, K. L. Miller, T. E. Nichols, E. C. Robinson, G. Salimi-Khorshidi, M. W. Woolrich, et al., "Functional connectomics from resting-state fmri," Trends in cognitive sciences 17, 666-682 (2013).

${ }^{51}$ S. H. Strogatz, Nonlinear dynamics and chaos with student solutions manual: With applications to physics, biology, chemistry, and engineering (CRC press, 2018).

${ }^{52}$ A. Palacios, "Heteroclinic cycles," Scholarpedia 2, 2352 (2007).

${ }^{53}$ I. Tsuda, "Chaotic itinerancy," Scholarpedia journal 8, 4459 (2013).
${ }^{54}$ A. Soong and C. Stuart, "Evidence of chaotic dynamics underlying the human alpha-rhythm electroencephalogram," Biological cybernetics 62, 5562 (1989).

${ }^{55}$ W. S. Pritchard and D. W. Duke, "Dimensional analysis of no-task human eeg using the grassberger-procaccia method," Psychophysiology 29, 182192 (1992).

${ }^{56}$ A. Babloyantz, J. Salazar, and C. Nicolis, "Evidence of chaotic dynamics of brain activity during the sleep cycle," Physics letters A 111, 152-156 (1985).

${ }^{57}$ A. Babloyantz and A. Destexhe, "Low-dimensional chaos in an instance of epilepsy," Proceedings of the National Academy of Sciences 83, 3513-3517 (1986).

${ }^{58}$ C. A. Skarda and W. J. Freeman, "How brains make chaos in order to make sense of the world," Behavioral and brain sciences 10, 161-173 (1987).

${ }^{59}$ H. Korn and P. Faure, "Is there chaos in the brain? ii. experimental evidence and related models," Comptes rendus biologies 326, 787-840 (2003).

${ }^{60} \mathrm{P}$. Faure and H. Korn, "Is there chaos in the brain? i. concepts of nonlinear dynamics and methods of investigation," Comptes Rendus de l'Académie des Sciences-Series III-Sciences de la Vie 324, 773-793 (2001).

${ }^{61}$ G. T. Einevoll, A. Destexhe, M. Diesmann, S. Grün, V. Jirsa, M. de Kamps, M. Migliore, T. V. Ness, H. E. Plesser, and F. Schürmann, "The scientific case for brain simulations," Neuron 102, 735-744 (2019).

${ }^{62}$ C. Letellier and O. E. Rossler, "Rossler attractor," Scholarpedia 1, 1721 (2006).

${ }^{63}$ S. Petkoski and V. K. Jirsa, "Transmission time delays organize the brain network synchronization," Philosophical Transactions of the Royal Society A 377, 20180132 (2019).

${ }^{64}$ P. Hagmann, L. Cammoun, X. Gigandet, R. Meuli, C. J. Honey, V. J. Wedeen, and O. Sporns, "Mapping the structural core of human cerebral cortex," PLoS Biol 6, e159 (2008).

${ }^{65}$ R. B. Berry, R. Brooks, C. E. Gamaldo, S. M. Harding, C. Marcus, B. V. Vaughn, et al., "The aasm manual for the scoring of sleep and associated events," Rules, Terminology and Technical Specifications, Darien, Illinois, American Academy of Sleep Medicine 176, 2012 (2012).

${ }^{66}$ N. Tzourio-Mazoyer, B. Landeau, D. Papathanassiou, F. Crivello, O. Etard, N. Delcroix, B. Mazoyer, and M. Joliot, "Automated anatomical labeling of activations in spm using a macroscopic anatomical parcellation of the mni mri single-subject brain," Neuroimage 15, 273-289 (2002).

${ }^{67}$ G. H. Glover, T.-Q. Li, and D. Ress, "Image-based method for retrospective correction of physiological motion effects in fmri: Retroicor," Magnetic Resonance in Medicine: An Official Journal of the International Society for Magnetic Resonance in Medicine 44, 162-167 (2000).

${ }^{68}$ D. Cordes, V. M. Haughton, K. Arfanakis, J. D. Carew, P. A. Turski, C. H. Moritz, M. A. Quigley, and M. E. Meyerand, "Frequencies contributing to functional connectivity in the cerebral cortex in "resting-state" data," American Journal of Neuroradiology 22, 1326-1333 (2001).

${ }^{69}$ R. Dosselmann and X. D. Yang, "A comprehensive assessment of the structural similarity index," Signal, Image and Video Processing 5, 81-91 (2011).

${ }^{70}$ Z. Wang, A. C. Bovik, H. R. Sheikh, and E. P. Simoncelli, "Image quality assessment: from error visibility to structural similarity," IEEE transactions on image processing 13, 600-612 (2004).

${ }^{71}$ A. Pikovsky, J. Kurths, M. Rosenblum, and J. Kurths, Synchronization: a universal concept in nonlinear sciences, Vol. 12 (Cambridge university press, 2003).

${ }^{72}$ J. C. Sprott, Chaos and time-series analysis, Vol. 69 (Citeseer, 2003).

${ }^{73}$ K. Xu, J. P. Maidana, S. Castro, and P. Orio, "Synchronization transition in neuronal networks composed of chaotic or non-chaotic oscillators," Scientific reports 8, 1-12 (2018).

${ }^{74}$ C. O. Fritz, P. E. Morris, and J. J. Richler, "Effect size estimates: current use, calculations, and interpretation." Journal of experimental psychology: General 141, 2 (2012).

${ }^{75} \mathrm{D}$. Kleppner and R. Kolenkow, An introduction to mechanics (Cambridge University Press, 2014).

${ }^{76}$ L. Abbott and T. B. Kepler, "Model neurons: from Hodgkin-Huxley to Hopfield," in Statistical mechanics of neural networks (Springer, 1990) pp. 518.

${ }^{77}$ S. W. Oh, J. A. Harris, L. Ng, B. Winslow, N. Cain, S. Mihalas, Q. Wang, C. Lau, L. Kuan, A. M. Henry, et al., "A mesoscale connectome of the mouse brain,” Nature 508, 207-214 (2014). 
${ }^{78}$ H. Markram, "The human brain project," Scientific American 306, 50-55 (2012).

${ }^{79}$ J. Courtiol, M. Guye, F. Bartolomei, S. Petkoski, and V. K. Jirsa, "Dynamical mechanisms of interictal resting-state functional connectivity in epilepsy," Journal of Neuroscience 40, 5572-5588 (2020).

${ }^{80}$ M. Schirner, A. R. McIntosh, V. Jirsa, G. Deco, and P. Ritter, "Inferring multi-scale neural mechanisms with brain network modelling," Elife 7, e28927 (2018).

${ }^{81} \mathrm{E}$. T. Rolls and G. Deco, The noisy brain: stochastic dynamics as a principle of brain function, Vol. 34 (Oxford university press Oxford, 2010).

${ }^{82}$ R. M. Hutchison, T. Womelsdorf, E. A. Allen, P. A. Bandettini, V. D. Calhoun, M. Corbetta, S. Della Penna, J. H. Duyn, G. H. Glover, J. GonzalezCastillo, et al., "Dynamic functional connectivity: promise, issues, and interpretations," Neuroimage 80, 360-378 (2013).

${ }^{83}$ A. A. Faisal, L. P. Selen, and D. M. Wolpert, "Noise in the nervous system," Nature reviews neuroscience 9, 292-303 (2008).

${ }^{84}$ A. Blumer, A. Ehrenfeucht, D. Haussler, and M. K. Warmuth, "Occam's razor," Information processing letters 24, 377-380 (1987).

${ }^{85} \mathrm{P}$. E. Rapp, "Is there evidence for chaos in the human central nervous system," in Chaos theory in psychology and the life sciences, edited by R. Robertson and A. Combs (Psychology Press, 2014).

${ }^{86} \mathrm{H}$. Preissl, W. Lutzenberger, and F. Pulvermüller, "Is there chaos in the brain?" Behavioral and Brain Sciences 19, 307-308 (1996).

${ }^{87} \mathrm{D}$. Hansel and H. Sompolinsky, "Synchronization and computation in a chaotic neural network," Physical Review Letters 68, 718 (1992).

${ }^{88}$ M. Rabinovich and H. Abarbanel, "The role of chaos in neural systems," Neuroscience 87, 5-14 (1998).

${ }^{89}$ J. L. Hindmarsh and R. Rose, "A model of neuronal bursting using three coupled first order differential equations," Proceedings of the Royal society of London. Series B. Biological sciences 221, 87-102 (1984).

${ }^{90} \mathrm{D}$. Hansel and H. Sompolinsky, "Chaos and synchrony in a model of a hypercolumn in visual cortex," Journal of computational neuroscience $\mathbf{3}$ 7-34 (1996).

${ }^{91}$ D. Battaglia, N. Brunel, and D. Hansel, "Temporal decorrelation of collective oscillations in neural networks with local inhibition and long-range excitation," Physical review letters 99, 238106 (2007).

${ }^{92}$ J. Kadmon and H. Sompolinsky, "Transition to chaos in random neuronal networks," Physical Review X 5, 041030 (2015).

${ }^{93}$ P. Orio, M. Gatica, R. Herzog, J. P. Maidana, S. Castro, and K. Xu, "Chaos versus noise as drivers of multistability in neural networks," Chaos: An Interdisciplinary Journal of Nonlinear Science 28, 106321 (2018).

${ }^{94}$ G. Deco, V. Jirsa, A. R. McIntosh, O. Sporns, and R. Kötter, "Key role of coupling, delay, and noise in resting brain fluctuations," Proceedings of the National Academy of Sciences 106, 10302-10307 (2009).

${ }^{95}$ S. Petkoski, J. M. Palva, and V. K. Jirsa, "Phase-lags in large scale brain synchronization: Methodological considerations and in-silico analysis," PLoS computational biology 14, e1006160 (2018).

${ }^{96}$ J. Cabral, H. Luckhoo, M. Woolrich, M. Joensson, H. Mohseni, A. Baker, M. L. Kringelbach, and G. Deco, "Exploring mechanisms of spontaneous functional connectivity in meg: how delayed network interactions lead to structured amplitude envelopes of band-pass filtered oscillations," Neuroimage 90, 423-435 (2014).

${ }^{97}$ T. Bayne and J. Hohwy, "Modes of consciousness," Finding consciousness: The neuroscience, ethics and law of severe brain damage , 57-80 (2016).

${ }^{98}$ J. Piccinini, "Awake time series," (2020). 